\title{
Relationships Among Motor Impairment, Gross Motor Function, and the ICF CY in Cerebral Palsy
}

\author{
Dong Baek Son ${ }^{1}$, Bo Young Hong ${ }^{2}$ and Kyoung Bo Lee ${ }^{3 *}$ \\ ${ }^{1}$ Center of Kidstory, 110 Jungbu-daero, Suwon-si, Republic of Korea \\ ${ }^{2}$ Department of Rehabilitation Medicine, St. Vincent's Hospital, College of Medicine, The Catholic University of Korea, Republic \\ of Korea
}

${ }^{3}$ Department of Physical therapy, St. Vincent's Hospital, College of Medicine, The Catholic University of Korea, Republic of Korea

*Corresponding author: Kyoung Bo Lee, Department of Physical Therapy, University of Catholic St. Vincent's Hospital, 93 Jungbudaero, Paldal-gu, Suwon-si, Republic of Korea

\section{ARTICLE INFO \\ Received: September 21, 2020}

Published: 幽 September 29, 2020

Citation: Dong Baek Son, Bo Young Hong, Kyoung Bo Lee. Relationships Among Motor Impairment, Gross Motor Function, and the ICF-CY in Cerebral Palsy. Biomed J Sci \& Tech Res 30(5)-2020. BJSTR. MS.ID.005012.

Abbreviations: CP: Cerebral Palsy; SEM: Structural Equation Modeling; WHO: World Health Organization; MTS: Modified Tardieu Scale; SMC: Selective Motor Control; GMFCS: Gross Motor Function Classification System; U/E: Upper Extremity; L/E: Lower Extremity; SMC: Selective Motor Control; GMF: Gross Motor Function

\section{ABSTRACT}

Purpose: To analyze relationships among motor impairment, gross motor function, and ICF-CY in children with cerebral palsy using a structural equation modeling.

Materials and Methods: The design was a cross-sectional study. Participants were 103(54 males, 49 females; mean age 9y2mo). Motor impairment(spasticity, muscle strength, selective motor control), gross motor function (GMFM-66), and Common Brief ICF-core set of CP were assessed.

Results: Confirmatory factor analysis for motor impairment showed that spasticity was more important than strength and selective motor control $(\beta=0.736, \mathrm{R} 2=54 \%)$. Motor impairment affected gross motor function $(\beta=0.869, \mathrm{p}<0.001)$ and explained $75 \%$ of the variance in variable. Gross motor function affected the ICF-CY $(\beta=-0.775, p$ $<0.001$ ) and explained $60 \%$ of the variance in variable. Motor impairment affected the ICF-CY indirectly $(\beta=-0.673, \mathrm{p}<0.001)$.

Conclusions: Motor impairments and gross motor function can predict gross motor function and ICF-CY levels, respectively.

Keywords: Cerebral palsy; Structural Equation Modeling; Motor impairment; gross motor function; ICF-CY

\section{Introduction}

Cerebral Palsy (CP), which refers to a group of permanent disorders in the development of movement and posture that limit activity, is attributed to non-progressive disturbances that occur in the developing fetal or infant brain. The motor disorders of CP are often accompanied by disturbances in sensation, cognition, communication, and behavior; by epilepsy; and by secondary musculoskeletal problems [1]. Manifestations of CP include spasticity, loss of selective motor control, muscle weakness, and limited range of motion, which further limit the performance of activities of daily living and participation in various activities [2]. In addition to disordered development of posture and movement, sufferers often have limitations in perception, cognition and communication and comorbidities that influence activity and participation. For example, preschool children with CP and poor motor function participated less in activities than those with better motor function [3]. When these impairments occur in the growing child, a reduced range of motion at a joint may also occur, creating abnormal biomechanical alignment that may further reduce function [4]. As a result of the increasing awareness of the importance of activities and participation arising from enablement models, such as the International Classification of Functioning, Disability and Health (ICF), functional outcomes have recently been 
emphasized in rehabilitation [5]. The ICF assists in understanding the importance of the relationship between participation in valued activities and roles and good health and well-being [6].

Fundamental goals of physical therapy for young children with $\mathrm{CP}$ are to optimize motor function, including adaptive function, prevent the development of secondary conditions, such as musculoskeletal deformities that impact lifelong health, and promote participation in daily life. Optimization of the motor outcomes of children with CP is an important area of investigation because of the associations of motor ability with self-care and play [7]. The study is supported by research on the relationship between motor impairment and gross motor function [8,9]. Spasticity is a major motor impairment in children with $\mathrm{CP}$ and negatively affects their activities; it also has a significant negative correlation with motor function [10]. Also, muscle weakness, which affects the degree of motor impairment, may be a determinant of the level of functioning, and several studies have shown that muscle strengthening is an important part of improving functional activity $[11,12]$. A multivariate analysis showed that selective motor control was the factor most strongly associated with the musculoskeletal functions examined $[9,10]$.

Previous results presented in terms of correlations have limitations for determining causal relationships between variables and explaining direct and indirect relationships $[9,13,14]$. Additionally, there is a lack of research that suggests a standardized structure with which to explain motor impairment. Thus, it is important to investigate the concept, definition, and validity of exercise injury through further studies. There have been some studies that used path analysis to show a relationship among exercise injuries, activities, and functional outcomes and further explored direct, indirect, and causal relationships among variables using Structural Equation Modeling (SEM) with factor analysis $[5,15]$. Research based on SEM is often used to identify complex causal relationships between factors and variables, as this approach reveals relationship between multiple dependent variables and independent variables simultaneously, enabling analyses from various perspectives. The World Health Organization (WHO) developed the ICF-core set to enhance the clinical use of this tool for specific diseases by including core items that fit a wide range of diseases [16]. The recently published ICF-core set can assess individual and environmental factors in children and adolescents with CP [17]. Thus, in this study, we examined the relationship between motor impairment and motor behavior through SEM and used these data as the basis to suggest the application of the ICF-CY (children and youths). The results of this study can provide a foundation for the development of therapeutic goals and intervention strategies. The purpose of this study was to use SEM to investigate direct and indirect relationships involving the ICF-CY, motor impairment, and gross motor function in children with $\mathrm{CP}$. We provide data for establishing therapeutic priorities, goals, and long-term intervention strategies.

\section{Materials and Methods}

\section{Participants}

This study used cross-sectional data collected from children with CP. Data were collected between August and October 2016. All children with diagnosed CP were eligible to participate in the study, conducted with children who were treated at general hospitals, welfare centers, and private institutions located in Seoul, Gyeong-gi, and Chung-nam provinces. One therapist assessed motor impairment, gross motor function, and the ICF-CY. The total time required was $20-30 \mathrm{~min}$, including break time, and the ICF-CY was administered during a parent counseling session. In cases of interruption during the evaluation process or in cases of fatigue, the remaining items were measured within 1 week. The total number of participants was 103 (Table 1), which fulfilled the criterion of 15 per observation variable [18] or at least 100 samples for research [19]. To evaluate several items, we selected children who could follow simple instructions. Ethics were obtained through the relevant ethics committees including The University of Yongin Ethics Committee(1607-HSR-054).

Table 1: General characteristics of participants $(\mathrm{N}=103)$.

\begin{tabular}{|c|c|c|}
\hline Characteristics & Number of Participants & $\%$ \\
\hline Sex (boy/girl) & $54 / 49$ & \\
Age (year) & $9.2 \pm 3.9$ & $52.4 / 47.6$ \\
Height (cm) & $127 \pm 19.7$ & \\
Weight (kg) & $30.5 \pm 14.1$ & 16.5 \\
\hline Type of cerebral palsy & 17 & 49.5 \\
Tetraplegia & 51 & 13.6 \\
Diplegia & 14 & 16.5 \\
Rt. Hemiplegia & 17 & $1.9 / 1.9$ \\
Lt. Hemiplegia & $2 / 2$ & 29.1 \\
Athetoid / Ataxia & & 27.2 \\
\hline GMFCS level & 30 & 12.6 \\
I & 28 & 31.1 \\
II & 13 & 0 \\
III & 32 & \\
IV & 0 & \\
V & & \\
\hline
\end{tabular}

Note: Values are mean \pm SD or $n(\%)$.

\section{Outcome Measures}

Motor Impairment: The Modified Tardieu Scale (MTS) was used to measure spasticity in the subjects. This instrument is effective for evaluating rigor and constriction and enables more accurate measurements than are possible with the Modified Ashworth Scale [20]. We measured the amount of muscle reaction that occurred at the highest speed in both shoulders, elbows, wrists, hips, knees, and ankles [21]. These scores are inversely related to the scores of other variables involved in motor impairment. Thus, we used inverse coding to transform all scores so that higher values 
indicate better function. The strength of each muscle was measured using a hand-held dynamometer (model 01163, Lafayette, IN, USA 2003). The measured muscles and their positions were selected with reference to previous studies [22,23]. After inducing the subject's movement, the evaluator performed the test, maintaining resistance at a specific location [24]. The maximum peak value was measured while holding for $3 \mathrm{~s}$ and was calculated as the average of the combined values of both sides after two measurements. To prevent muscle fatigue during each measurement, a rest period of 45s was provided [25], and a pelvic belt was used for stability [26]. The reliability of the test for nervous system patients has been reported to be as high as 0.84 to 0.94 [27].

The peak force value for each muscle was converted to Newtons, and the sum of the upper and lower limb groups was normalized [28]. Selective Motor Control (SMC) is the ability to move an individual joint independently from posture and other joint movements in the same limb [21]. To assess SMC, the children were asked to perform dorsiflexion of the ankle and extension of the knee of each leg in the sitting position on an examination table while the occurrence of synergistic movements in the other joints was observed. Test-retest reliability ranged from 0.88 to 1.00 [29].

Gross Motor Function: Gross motor function was measured using the GMFM-66 [30]. This is a standardized observational instrument designed to measure gross motor capacity in children and youth with CP who are aged 0 to 18 years. The GMFM-66 includes five groups of items, with 66 items in total. Test-retest reliability has been reported to be as high as 0.99 [31]. Items were estimated using the Gross Motor Ability Estimator program [32].

ICF-CY(Children and Youth): The ICF-CY was based on the observation that derivative classes are needed to explain the health status of children and adolescents in particular [33]. However, it was difficult to use in clinical situations because many areas and items are included in the evaluation [17]. WHO developed ICF-CY core sets were developed for each age group. The recently published ICF-core set for children with $\mathrm{CP}$ is divided into Comprehensive, Common Brief, and three age-specific Core set areas [17]. Among them, the Common Brief ICF-core set consists of one body structure, eight body composition, eight activity, eight participation, and eight environment items for 0-18-year-olds. In this study, the 17 items that did not address the environmental factors were evaluated.

\section{Data Analysis}

This was a cross-sectional study of children with CP. The SPSS (ver. 18.0) and AMOS (ver. 18.0) software packages were used for the analysis of descriptive statistics and SEM. The most commonly used maximum likelihood method for model estimation was used; this is a method for obtaining the highest probability of occurrence through observed data. SEM was used to examine relationships among motor impairment, gross motor function, and the ICF-CY in children with CP and to investigate the roles played by motor impairment and gross motor function in mediating these relationships or in directly predicting functional outcomes.

\section{Results}

We examined the normality of the observation variables for skewness and kurtosis (Table 2). It has been shown that skewness regularity is not satisfied in spasticity, upper extremity muscle strength, or lower extremity muscle strength. Kurtosis regularity is not satisfied in upper extremity muscles or lower extremity muscles. On this basis, it may be necessary to confirm the adequacy of the model through other fitness indices. We also examined whether there was a problem with multicollinearity given the possible correlations between variables. Upper extremity muscle strength showed no significant correlation with SMC, spasticity, or gross motor function (Table 3). The significance of the relationships involving the remaining variables did not exceed 0.90. Confirmatory factor analysis was performed to generate a latent variable for motor impairment (Figure. 1). The lower extremity factor loading was slightly lower, at 0.48 . The path coefficients of spasticity, lower extremity strength, and SMC, representing motor impairment, were all significant (Table 4). The highest factor was spasticity $(\beta=0.736$, R2 $=54 \%$ ). Additionally, the path coefficient and the coefficient of determination (R2) between motor impairment and gross motor function were high $(\beta=0.869, \mathrm{R} 2=75 \%)$. The strong correlation between the ICF-CY and gross motor function yielded a coefficient of determination greater that $50 \%(\beta=-0.775, \mathrm{R} 2=60 \%)$, and the critical ratio of most variables exceeded \pm 1.965 . The indirect effect of the ICF-CY pathway on motor impairment was $0.673(\mathrm{p}<0.001)$. The proposed path model used for this study is shown in Figure. 2. Model fit is expressed as absolute fit index and incremental fit index. In the absolute fit indices, $\chi^{2}$ and RMSEA were 4.293 ( $p>$ 0.05 ) and $<0.001$, respectively. The incremental fit indices NFI, CFI, and TLI were $0.981,1.000$, and 1.007 , respectively.

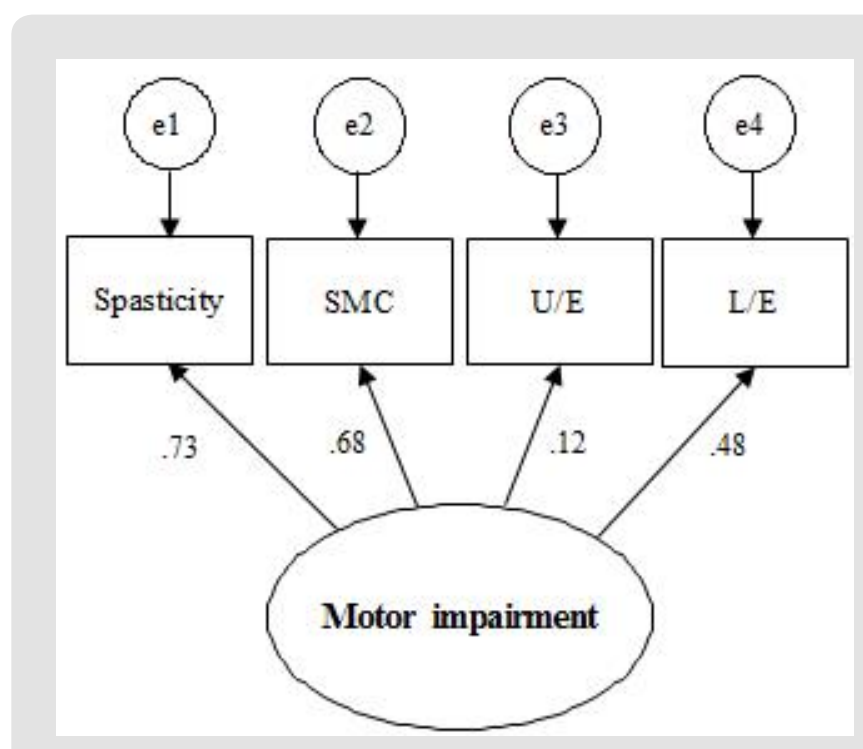

Figure 1: Confirmatory factor analysis of study model. 
Table 2: Descriptive statistics of the observed variables(N=103).

\begin{tabular}{|c|c|c|c|c|}
\hline & Skewness & CR & Kurtosis & CR \\
\hline Spasticity & -0.827 & -3.426 & 0.558 & 1.156 \\
U/E & 1.72 & 7.125 & 6.826 & 4.723 \\
L/E & 1.12 & 4.64 & 2.28 & -1.898 \\
SMC & -0.055 & -0.229 & -0.916 & -2.604 \\
GMF & 0.012 & 0.049 & -1.257 & -1.367 \\
ICF-CY & 0.315 & 1.305 & -0.66 & \\
\hline
\end{tabular}

Table 3: Correlations between the observed variables(N=103).

\begin{tabular}{|c|c|c|c|c|c|}
\hline & Spasticity & U/E & L/E & SMC & \\
\hline Spasticity & & & & \\
U/E & 0.022 & $.745^{* *}$ & & \\
L/E & $.280^{* *}$ & -0.038 & $.271^{* *}$ & $.585^{* *}$ \\
SMC & $.549^{* *}$ & 0.061 & $.444^{* *}$ & $-.465^{* *}$ \\
GMF & $.633^{* *}$ & -0.115 & $-.351^{* *}$ & $-.775^{* *}$ \\
ICF-CY & $-.539^{* *}$ & & & \\
\hline
\end{tabular}

Note: *Significant at $\mathrm{P}<.05,{ }^{* *}$ Significant at $\mathrm{P}<.01$.

Table 4: Path coefficients of the study model.

\begin{tabular}{|c|c|c|c|c|}
\hline Path & $\boldsymbol{\beta}^{\text {a }}$ & Standard error & Critical ratio & p \\
\hline Motor impairment $\rightarrow$ Spasticity & 0.736 & & & $* .266$ \\
Motor impairment $\rightarrow$ L/E & 0.461 & 0.04 & 6.328 & $* * *$ \\
Motor impairment $\rightarrow$ SMC & 0.688 & 0.1 & 6.898 & $* * *$ \\
Motor impairment $\rightarrow$ GMF & 0.869 & 0.824 & -12.383 & $* * *$ \\
GMF $\rightarrow$ ICF-CY & -0.775 & 0.04 & $*$ \\
\hline
\end{tabular}

Note: ${ }^{a}$ Significant at Standard coefficients, ${ }^{* * *}$ Significant atP $<.001$.

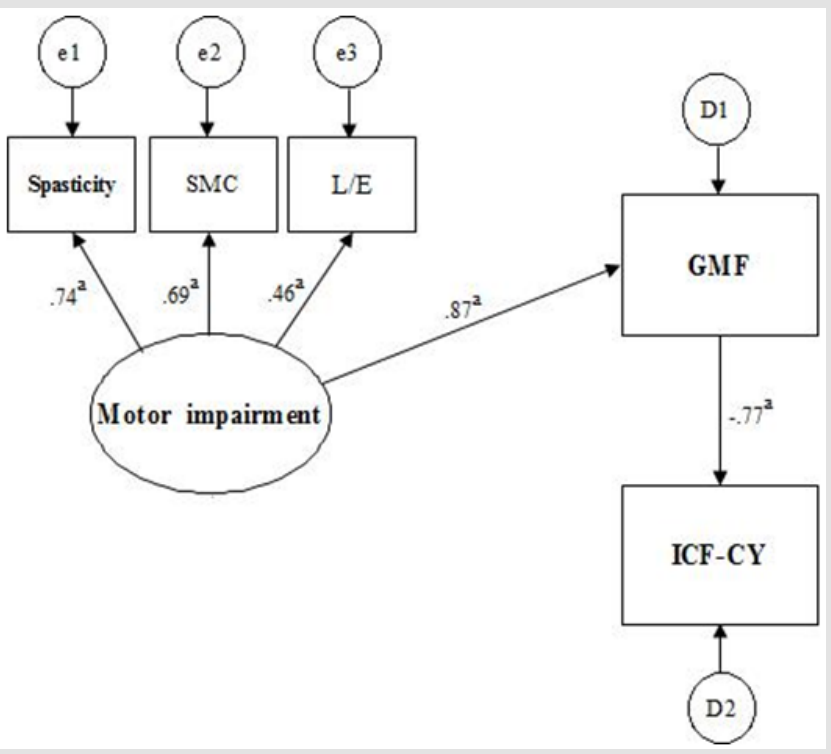

Figure 2: Structural equation modeling of study model.

\section{Discussion}

The goal for children with CP is improvement in the damaged motor functions. Furthermore, final goal is improvement in function-related quality of life. Quality of life is affected by the degree of personal motor impairment, psychological state, and ability to perform the activities of daily living [34]. This is why many studies have provided evidence for the effects of impaired motor function, gross motor function, and functional performance in children with CP $[5,10,13]$. In this study, we also examined the roles of the ICF$\mathrm{CY}$ and gross motor function by measuring the various aspects of motor impairment and identifying particularly important factors through confirmatory factor analysis. The path coefficients and the coefficient of determination were as follows: spasticity $(\beta=0.736$ and R2 $=54 \%)$, SMC $(\beta=0.688$ and R2 $=47 \%)$, lower extremity strength $(\beta=0.461$ and $\mathrm{R} 2=21 \%)$, gross motor function $(\beta=0.869$ and R2 $=75 \%)$, and the ICF-CY ( $\beta=-0.775$ and R2 $=60 \%)$. In this study, spasticity, motor control, and lower extremity strength were the main factors affecting motor impairment. 
Spasticity and SMC were relatively important, whereas the coefficient of determination for the impact of lower extremity strength was low. The factor with the greatest importance for motor impairment was spasticity. Spasticity can cause problems in daily activities, such as walking, eating, and dressing; over time, pain, spasms, and limitations in movement (e.g., sitting, and standing) can develop, which, in turn, affect joint contracture and abnormal body alignment [35]. Spasticity may also have a positive role in the standing position, where it induces the activation of leg and trunk extensors and maintains the size of muscles and bone density [36]. In this study, spasticity was the main factor affecting motor impairment, and the same result was reported in another study [5]. In another study, SMC had a major impact on gross motor function [9]. In this study, the path coefficient of SMC was high, reflecting its importance for forecasting and determining the level of gross motor function. Clinically, we can set goals through the assessment of SMC. Muscle weakness is one clinical symptom of CP, and it is also observed in children walking without help. In particular, the lower extremity muscles of children with $\mathrm{CP}$ are weaker, by 6-59\%, compared with those of peers [37]. Also, weakness of the distal muscles and muscular strength differences in agonistic and antagonistic muscles may also be present [38].

This affects functional positioning, including sitting and standing, as well as locomotor function, including walking [39]. In this study, we also evaluated lower extremity muscle strength. The path coefficient and the coefficient of determination (R2) of lower extremity muscle strength were relatively low compared with those of the other factors tested. Another study reported that muscle strength did not affect the activity and participation of children with CP [40]. Children with CP showed a decrease in muscle strength over time, and a smaller decrease was seen in children with independent gait [38]. This is likely a result of the correlation between walking and strength; other factors beyond decreasing muscle strength may have effects as children age. It has also been reported that adaptive behavior, rather than muscle strength, increases self-motivation and participation opportunities, leading to improved motor function [13]. However, it can be seen that measuring the prognosis of children only by determining their physical characteristics is difficult. Further research in this area is needed.

Upper extremity muscle strength is an important contributor to limitations in daily life. Grip strength is a good predictor of hand manipulation activities in the context of damaged upper extremities [41]. However, confirmatory factor analysis of motor impairment yielded a low path coefficient (Figure 1). There was also no significant correlation with other variables tested, and it was hard to conceptualize upper extremity muscle strength as an appropriate factor to explain motor impairment [42]. This may be attributable to the number of diplegia and hemiplegia cases included in our sample, as these individuals seemed a little slower at manipulating things but were still able to perform. Additionally, other studies have reported a significant difference between lower limb strength and gross motor function versus upper extremity strength $[14,43]$. It has been reported that the effects on the sitting and standing functions are not due to upper extremity strength but to the strength of the extensors of the knee and trunk [14].

This study found that motor impairment had a major effect on gross motor function, and the coefficient of determination in this regard was high. Gross motor function-related motor impairment is goal of interventions for CP children with associated motor impairment. It is also a factor influencing social participation and activities [14]. A recent study showed that motor impairment had major effects on gross motor function and on functional outcomes [5]. Children with higher levels of gross motor function will have more opportunities for activity and participation [3]. The ICF-CY used here includes the ability to perform activities in everyday life, communicate with others, and participate in school and the community. The recently published ICF-core set can be applied to children with $\mathrm{CP}$, which is specifically included, and can be administered in short interviews [17]. In this study, the effect of gross motor function on the ICF-CY and the coefficient of determination were high. This is similar to the high path coefficients for gross motor function and functional outcome [5]. Although based on different evaluation methods, these results are consistent with those related to aspects of self-management, mobility, and social function. Additionally, this study found indirect effects of motor impairment and the ICF-CY(-.673). The degree of the physical activity of children with CP is an important factor influencing their quality of life and level of participation in everyday life; it also changes according to the severity of the disease [38]. This study found that motor impairment affected gross motor function and indirectly affected the ICF CY. Based on the results of this study, it is important to maximize current capabilities and seek future directions for interventions.

The limitations of this study include our inability evaluate the children with $\mathrm{CP}$ in a way that was more appropriate for the research method, which would have entailed increasing the number of subjects to increase the normality and fitness of the data and to enable the model to more closely represent the actual population. Additionally, as $\sim 50 \%$ of the subjects were diplegia, the application of our results to children with other categories of $\mathrm{CP}$ will be limited. Another limitation was the difficulty of assessing the muscle strength in the selected joint or to evaluate the muscle tension as it increased. In this context, it has been proposed that the level of activity be assessed using muscle forces rather than the strength of each muscle when measuring the effects of interventions [44]. Additionally, the ICF-core set was used as an evaluation tool; to date, however, no other reported study in Korea has used this set, rendering it difficult to compare this with other studies. Finally, the path analysis of muscular strength and gross motor function in each 
muscle group did not confirm a more specific causal relationship. Further studies are needed to determine which muscle group is most important for posture and function in clinical practice.

\section{Conclusion}

We examined direct and indirect relationships among motor impairment, gross motor function, and the ICF-CY. Motor impairment affected gross motor function and indirectly affected the ICF-CY. It was also confirmed that the ICF-CY had a direct effect. Based on these results, we can prioritize goals and long-term intervention strategies for the early treatment of children with CP. Additionally, understanding and studying the lives of children with cerebral palsy will increase our ability to support pediatric therapists to assist children in finding and fulfilling roles in the community.

\section{Conflict of Interest}

The authors have declared no conflicts of interest.

\section{References}

1. Rosenbaum P, Paneth N, Leviton A, Goldstein M, Bax M, et al. (2007) A report: the definition and classification of cerebral palsy April 2006. Developmental medicine and child neurology Supplement 109: 8-14.

2. Calley A, Williams S, Reid S, Blair E, Valentine J, et al. (2012) A comparison of activity, participation and quality of life in children with and without spastic diplegia cerebral palsy. Disability and rehabilitation 34(15): 1306-1310.

3. Law M, King G, Petrenchik T, Kertoy M, Anaby D (2012) The assessment of preschool children's participation: internal consistency and construct validity. Physical \& occupational therapy in pediatrics 32(3): 272-287.

4. Sukal Moulton T, Clancy T, Zhang LQ, Gaebler-Spira D (2014) Clinical application of a robotic ankle training program for cerebral palsy compared to the research laboratory application: does it translate to practice? Archives of physical medicine and rehabilitation 95(8): 14331440.

5. Park EY, Kim WH (2013) Structural equation modeling of motor impairment, gross motor function, and the functional outcome in children with cerebral palsy. Research in developmental disabilities 34(5): 1731-1739.

6. World Health Organization (2001). The International Classification of Functioning, Disability and Health (ICF). Geneva.

7. Chiarello LA, Palisano RJ, Bartlett DJ, Mc Coy SW (2011) A multivariate model of determinants of change in gross-motor abilities and engagement in self-care and play of young children with cerebral palsy. Physical \& occupational therapy in pediatrics 31(2): 150-168.

8. Hanna SE, Rosenbaum PL, Bartlett DJ, Palisano RJ, Walter SD, et al. (2009) Stability and decline in gross motor function among children and youth with cerebral palsy aged 2 to 21 years. Developmental medicine and child neurology 51(4): 295-302.

9. Vos RC, Becher JG, Voorman JM, Gorter JW, Van Eck M, et al. (2016) Longitudinal Association Between Gross Motor Capacity and Neuromusculoskeletal Function in Children and Youth With Cerebral Palsy. Archives of physical medicine and rehabilitation 97(8): 13291337.

10. Ostensjø S, Carlberg EB, Vøllestad NK (2004) Motor impairments in young children with cerebral palsy: relationship to gross motor function and everyday activities. Developmental medicine and child neurology 46(9): 580-589.
11. Dodd KJ, Taylor NF, Damiano DL (2002) A systematic review of the effectiveness of strength-training programs for people with cerebral palsy. Archives of physical medicine and rehabilitation 83(8): 11571164.

12. Mockford M, Caulton JM (2008) Systematic review of progressive strength training in children and adolescents with cerebral palsy who are ambulatory. Pediatric physical therapy : the official publication of the Section on Pediatrics of the American Physical Therapy Association $20(4): 318-333$

13. Bartlett DJ, Chiarello LA, Mc Coy SW, Palisano RJ, Jeffries L, et al. (2014) Determinants of gross motor function of young children with cerebral palsy: a prospective cohort study. Developmental medicine and child neurology 56(3): 275-282.

14. Ohata K, Tsuboyama T, Haruta T, Ichihashi N, Kato T (2008) Relation between muscle thickness, spasticity, and activity limitations in children and adolescents with cerebral palsy. Developmental medicine and child neurology 50(2): 152-156.

15. Kim WH, Park EY (2011) Causal relation between spasticity, strength, gross motor function, and functional outcome in children with cerebral palsy: a path analysis. Developmental medicine and child neurology 53(1): 68-73.

16. World Health Organization (2012). ICF core sets: Manual for clinical practice. Geneva.

17. Schiariti V, Klassen AF, Cieza A, Sauve K, O Donnell M, et al. (2014) Comparing contents of outcome measures in cerebral palsy using the International Classification of Functioning (ICF-CY): a systematic review. European journal of Paediatric neurology : EJPN : official journal of the European Paediatric Neurology Society 18(1): 1-12.

18. Stevens J (1996) Applied multivariate statistics for the social sciences Mahwah, NJ, Lawrence Erlbaum Associates.

19. Hair JF, Black WC, Babin BJ, Anderson RE, Tatham R (2006) Multivariate data analysis . Uppersaddle River NJ: Pearson Prentice Hall.

20. Alhusaini AA, Dean CM, Crosbie J, Shepherd RB, Lewis J (2010) Evaluation of spasticity in children with cerebral palsy using Ashworth and Tardieu Scales compared with laboratory measures. Journal of child neurology 25(10): 1242-1247.

21. Boyd RN, Graham HKJEJoN (1999) Objective measurement of clinical findings in the use of botulinum toxin type A for the management of children with cerebral palsy. 6: s23-s35.

22. Andrews AW, Thomas MW, Bohannon RW (1996) Normative values for isometric muscle force measurements obtained with hand-held dynamometers. Physical therapy 76(3): 248-259.

23. Van der Ploeg RJ, Fidler V, Oosterhuis HJ (1991) Hand-held myometry: reference values. Journal of neurology, neurosurgery, and psychiatry 54(3): 244-247.

24. Hébert LJ, Maltais DB, Lepage C, Saulnier J, Crête M (2015) Hand-Held Dynamometry Isometric Torque Reference Values for Children and Adolescents. Pediatric physical therapy : the official publication of the Section on Pediatrics of the American Physical Therapy Association 27(4): 414-423.

25. Dallmeijer AJ, Baker R, Dodd KJ, Taylor NF (2011) Association between isometric muscle strength and gait joint kinetics in adolescents and young adults with cerebral palsy. Gait \& posture 33(3): 326-332.

26. Bohannon RWJI, Science E (2012) Hand-held dynamometry: a practicable alternative for obtaining objective measures of muscle strength 20(4): 301-315.

27. Bohannon RW, Andrews AW (1987) Interrater reliability of hand-held dynamometry. Physical therapy 67(6): 931-933.

28. Wiley ME, Damiano DL (1998) Lower-extremity strength profiles in spastic cerebral palsy. Developmental medicine and child neurology 40(2): 100-107. 
29. Löwing K, Brogren Carlberg EJAiP (2009) Reliability of the selective motor control scale in children with cerebral palsy 11(2): 58-63.

30. Alotaibi M, Long T, Kennedy E, Bavishi S (2014) The efficacy of GMFM-88 and GMFM-66 to detect changes in gross motor function in children with cerebral palsy (CP): a literature review. Disability and rehabilitation 36(8): 617-627.

31. Russell DJ, Avery LM, Rosenbaum PL, Raina PS, Walter SD, et al. (2000) Improved scaling of the gross motor function measure for children with cerebral palsy: evidence of reliability and validity. Physical therapy 80(9): 873-885.

32. Russell DJ, Rosenbaum P, Wright M, Avery LM (2002) Gross motor function measure (GMFM-66 \& GMFM-88) user's manual.

33. Gan SM, Tung LC, Yeh CY, Wang CH (2013) ICF-CY based assessment tool for children with autism. Disability and rehabilitation 35(8): 678-685.

34. Jaspers E, Verhaegen A, Geens F, Van Campenhout A, Desloovere K, et al. (2013) Lower limb functioning and its impact on quality of life in ambulatory children with cerebral palsy. European journal of Paediatric neurology : EJPN : official journal of the European Paediatric Neurology Society $17(6):$ 561-567.

35. Lundy C, Lumsden D, Fairhurst C (2009) Treating complex movement disorders in children with cerebral palsy. The Ulster medical journal 78(3): 157-163.

36. Meythaler JM (2001) Concept of spastic hypertonia. Physical medicine and rehabilitation clinics of North America 12(4): 725-732.

37. Eek MN, Beckung E (2008) Walking ability is related to muscle strength in children with cerebral palsy. Gait \& posture 28(3): 366-371.

38. Davids JR, Oeffinger DJ, Bagley AM, Sison Williamson M, George Gorton (2015) Relationship of Strength, Weight, Age, and Function in Ambulatory Children With Cerebral Palsy. Journal of pediatric orthopedics 35(5): 523-529.

39. Ferland C, Lepage C, Moffet H, Maltais DB (2012) Relationships between lower limb muscle strength and locomotor capacity in children and adolescents with cerebral palsy who walk independently. Physical \& occupational therapy in pediatrics 32(3): 320-332.

40. Oeffinger D, Gorton G, Hassani S, Sison-Williamson M, Johnson B, et al (2014). Variability explained by strength, body composition and gait impairment in activity and participation measures for children with cerebral palsy: a multicentre study. Clinical rehabilitation 28(10): 10531063.

41. Klingels K, Demeyere I, Jaspers E, De Cock P, Molenaers G, et al. (2012) Upper limb impairments and their impact on activity measures in children with unilateral cerebral palsy." European journal of paediatric neurology : EJPN : official journal of the European Paediatric Neurology Society 16(5): 475-484

42. Sakzewski L, Ziviani J, Boyd R (2010) The relationship between unimanual capacity and bimanual performance in children with congenital hemiplegia. Developmental medicine and child neurology 52(9): 811-816

43. Thompson N, Stebbins J, Seniorou M, Newham D (2011) Muscle strength and walking ability in diplegic cerebral palsy: implications for assessment and management. Gait \& posture 33(3): 321-325.

44. Scianni A, Butler JM, Ada L, Teixeira Salmela LF (2009) Muscle strengthening is not effective in children and adolescents with cerebral palsy: a systematic review. The Australian journal of physiotherapy 55(2): 81-87.
ISSN: 2574-1241

DOI: $10.26717 /$ BJSTR.2020.30.005012

Kyoung Bo Lee. Biomed J Sci \& Tech Res

(C) This work is licensed under Creative

Submission Link: https://biomedres.us/submit-manuscript.php ommons Attribution 4.0 License

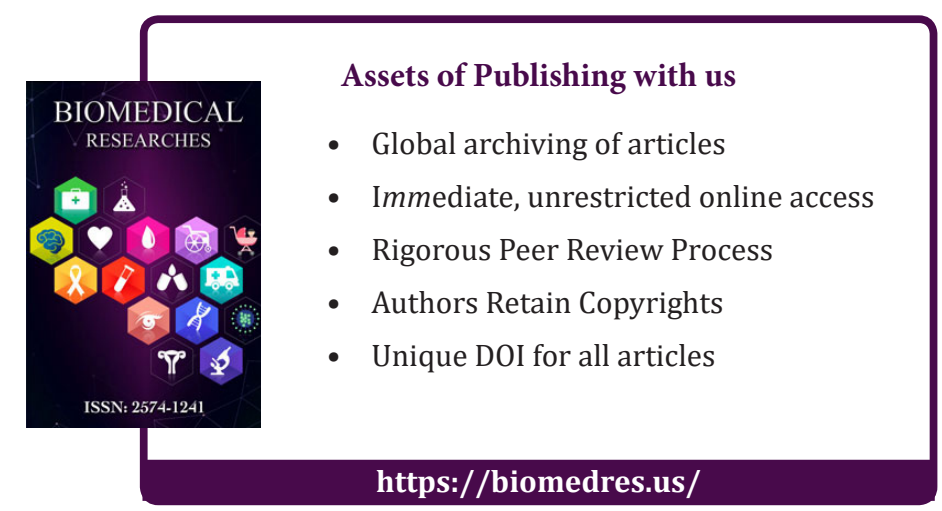

